\title{
O PROCESSO DE DESENVOLVIMENTO DE PRODUTOS VIA CROWD- DESIGN: oportunidades para a sustentabilidade
}

\author{
DICKIE, Isadora Burmeister. \\ PPGDesign/UFPR \\ isadora.dickie@gmail.com \\ SANTOS, Aguinaldo dos. \\ PPGDesign/UFPR \\ asantos@ufpr.br \\ BOTTANELLI, Greta. \\ Politécnico de Milão/Itália \\ greta.bottanelli@mail.polimi.it \\ CUCCU, Michele. \\ Politécnico de Milão/Itália \\ mikkk@live.it
}

Resumo: O presente artigo avalia as implicações para a sustentabilidade do processo de desenvolvimento de produtos realizados em plataformas de Crowd-Design. O texto é baseado em pesquisa-ação em andamento no Núcleo de Design e Sustentabilidade da UFPR, através do Projeto Sustainability Maker. Com base na literatura e nos resultados obtidos com a realização da primeira etapa do estudo em andamento, é apresentada uma análise comparativa do modelo ortodoxo do processo de desenvolvimento de produtos com um modelo baseado no Crowd-Design, incluindo recomendações para aplicações práticas bem como as principais vantagens e desvantagens desta abordagem de projeto sob a ótica da sustentabilidade.

Palavras-chave: inovação, PDP, Crowd-Design, design para a sustentabilidade.

Abstract: This paper assesses the implications for the sustainability of the
product development process performed in Crowd-Design platforms. The
text is based on action research in progress at the <blind review> through
Sustainability Maker Project. It presents also a comparative analysis of the
orthodox model of the product development process with a model based on
Crowd-Design, based on the literature and the results obtained by carrying
out the first stage of the ongoing study. It includs recommendations for 
practical applications as well as main advantages and disadvantages of this project from the perspective of sustainability approach.

Keywords: innovation, DPP, Crowd-Design, design for sustainability

\section{INTRODUÇÃO}

O contexto da rápida expansão da conectividade em rede oferece grandes oportunidades para avanços no campo do Design, especificamente no processo de desenvolvimento de produtos (PDP). Dentre as oportunidades está o fenômeno da crescente abertura do PDP para a participação de todos os stakeholders através da internet, configurando uma verdadeira "multidão".

Conforme Tapscott \& Williams (2008) a colaboração em massa, através de seu custo reduzido, permitem que muitos milhares de indivíduos e pequenos produtores criem em conjunto soluções em produtos/serviços e ingressem em mercados, o que no passado só as grandes empresas conseguiam. Desta forma, tem-se como pressuposto que o envolvimento desta multidão no processo de Design (Crowd-Design) pode gerar não só um número maior de soluções alternativas, mas também reduzir o tempo de desenvolvimento de projetos, diminuir custos, além de possibilitar a criação de redes de suporte à implementação e manutenção das soluções.

Este artigo se propõe a avaliar as implicações para a sustentabilidade do processo de desenvolvimento de produtos realizados em plataformas de Crowd-Design a partir de uma análise comparativa entre o modelo ortodoxo do processo de desenvolvimento de produtos e um modelo baseado no Crowd-Design. O tema vem sendo analisado no âmbito do Núcleo de Design e Sustentabilidade da UFPR através do Projeto Sustainability Maker (www.sustainabilitymaker.org). O projeto é liderado pela empresa E-Concept (Alemanha), sendo o desenvolvimento da plataforma financiado pela Comunidade Européia através do Programa LIFE (LIFE11 ENV/DE/000342). No projeto Sustainability Maker um dos autores deste artigo ocupa a posição no Advisory Board.

\section{DEFINIÇÕES}

O Crowd-Design é uma modalidade emergente de sistema de projeto e produção que utiliza os conhecimentos e recursos disponíveis na multidão, geralmente através da internet, com o propósito de resolver problemas e/ou criar conteúdo. Sua realização pode ocorrer de forma voluntária ou remunerada.

Outra abordagem associada ao Crowd-Design é o Crowd-Sourcing. Este último é um termo utilizado pela primeira vez em 2006, por Jeff Howe em um artigo da revista Wired (ALONSO, 2012; BAYUS, 2013 e ABRAHAMSON, 2013). Crowd-Sourcing consiste no ato de terceirizar, de maneira aberta e colaborativa, um trabalho tradicionalmente realizado por um funcionário contratado de uma empresa. Esta terceirização acontece sob a forma de um convite aberto para um grande grupo de pessoas, e geralmente é realizado a partir da web.

O processo de seleção de alternativas nestes ambientes voltados à multidão pode utilizar o Crowd-Voting. Este usualmente ocorre através de sites que buscam obter a opinião de um grande número de pessoas acerca de um determinado tópico. Exemplo de Crowd-Voting pode ser encontrado na votação sobre qual a melhor 
tradução para um dado texto na plataforma Mechanical Turk, da Amazon (ALONSO E MIZZARRO, 2012; ALONSO, 2012; AZZAM E JACOBSON, 2013; BANNERMMAN, 2013).

Para financiar projetos através de envolvimento da multidão pode-se utilizar o Crowd-Funding, que consiste da solicitação pública de financiamento de projetos específicos. Nesta abordagem, contribuições pequenas de indivíduos isolados são somadas de forma a contribuir para viabilizar a execução de um dado projeto (ROBSON, 1993). O financiamento é solicitado on-line, geralmente em quantidades relativamente pequenas, a partir de doadores individuais ou investidores, e são destinadas para projetos específicos, como: empréstimos pessoais para as pequenas empresas, produção camisetas (t-shirt), ou a produção de filmes ou música (BANNERMAN, 2013). Nesse sentido, o Crowd-Funding desafia o modelo tradicional de investimento privado, através do qual um grande investidor ou um pequeno grupo de investidores financia um projeto (BELLEFLAMME et al., 2011). O financiamento pode ocorrer também através de maneira similar a equity funds, onde os apoiadores passam a ser sócios do projeto.

\section{MODELOS DO PROCESSO DE DESENVOLVIMENTO DE PRODUTOS (PDP)}

\subsection{Modelos Ortodoxos do PDP}

Gomes (2011) comparou os modelos de Processo de Desenvolvimento de Produtos (PDP) propostos por Crul e Diehl (2006), Charter e Tischner (2001), Varžinskas (2007), Pereira (2003), Rozenfeld et al. (2006) e Baxter (2000). O autor conclui que o modelo de Rozenfeld et al. (2006) é o mais completo dentre estes autores. Sua estrutura é baseada em três macro-fases: pré-desenvolvimento, desenvolvimento e pós-desenvolvimento, conforme descrito a seguir.

Na macro-fase de pré-desenvolvimento é onde é delineada a estratégia de PDP da empresa, incluindo os objetivos e metas relativos ao portfólio. Esta macro-fase divide-se em Planejamento Estratégico do Produto e Planejamento do Projeto. 0 objetivo do Planejamento Estratégico do Produto é a definição de um portfólio de produtos para a empresa, ou seja, descrever uma linha de produtos e os projetos a serem desenvolvidos. Este Planejamento Estratégico pode envolver inclusive planos de retiradas de produtos já constantes no portfólio. O Planejamento do Projeto é um elemento essencial desta macro-fase, apontando desde o escopo do projeto e do produto, orçamento, prazos, definição do pessoal, recursos, procedimentos de avaliação, análises de risco e indicadores de desempenho do projeto e produto (ROZENFELD et al., 2006).

A macro-fase de desenvolvimento envolve o processo de projeto, detalhamento e produção do produto. Esta macro-fase divide-se em Projeto Informacional, Projeto Conceitual, Projeto Detalhado, Preparação da Produção e Lançamento do produto. Na fase de do Projeto Informacional devem resultar as especificações-meta do produto, que orientarão a geração de soluções, fornecendo uma base para elaboração de critérios de avaliação e tomadas de decisão. A fase seguinte trata do Projeto Conceitual, o qual objetiva a busca, criação, representação e seleção de soluções para o problema identificado no Projeto Informacional. No Projeto Detalhado, são realizadas todas as especificações e detalhamentos, os protótipos são testados resultando no detalhamento de todos os recursos, manuais de uso, instruções de assistência, além de suporte às equipes de venda. Finalmente, na Preparação da Produção é produzido um lote de produção piloto, permitindo que o 
processo produtivo seja mapeado e melhor definido. Por fim, ocorre o Lançamento do Produto, implicando em projeto e implementação de processos de assistência técnica e auxílio ao consumidor (ROZENFELD et al., 2006).

Finalmente, a última macro-fase do modelo de Rozenfeld et al (2006) trata do pós-desenvolvimento, que inclui as atividades do pós venda até o fim do seu ciclo de vida do produto. Esta macro-fase divide-se no modelo nas etapas de Acompanhar o Produto e Processo e Descontinuar o Produto. O acompanhamento do produto inclui a realização de atividades como: avaliação da satisfação do cliente, o monitoramento do desempenho do produto, auditoria pós-projeto e o registro de lições aprendidas. A descontinuidade do produto inclui as atividades de: analisar e aprovar descontinuidade do produto, planejar a descontinuidade do produto, preparar o recebimento do produto, acompanhar o recebimento do produto, descontinuar a produção, finalizar suporte ao produto e a avaliação geral e encerramento do projeto (ROZENFELD et al., 2006).

\subsection{Modelo de PDP via Crowd-Design}

O modelo de PDP via Crowd-Design apresentado na figura a seguir é adotado no Projeto Sustainability Maker (SuM, 2014, web). Neste modelo, o ponto de partida refere-se à compreensão dos problemas de uma dada comunidade ou organização, bem como suas implicações para a sustentabilidade. Com o propósito de determinar o problema que seja efetivamente relevante na percepção da comunidade/organização afetada, estes são votados pelo próprio grupo de pessoas que os relatou. Acontece, então, o primeiro Crowd-Voting. A partir daí, é definido o desafio - etapa challenge -, cujo processo de desenvolvimento da ideia solução é aberto e realizado a partir do processo de Crowd-Sourcing.

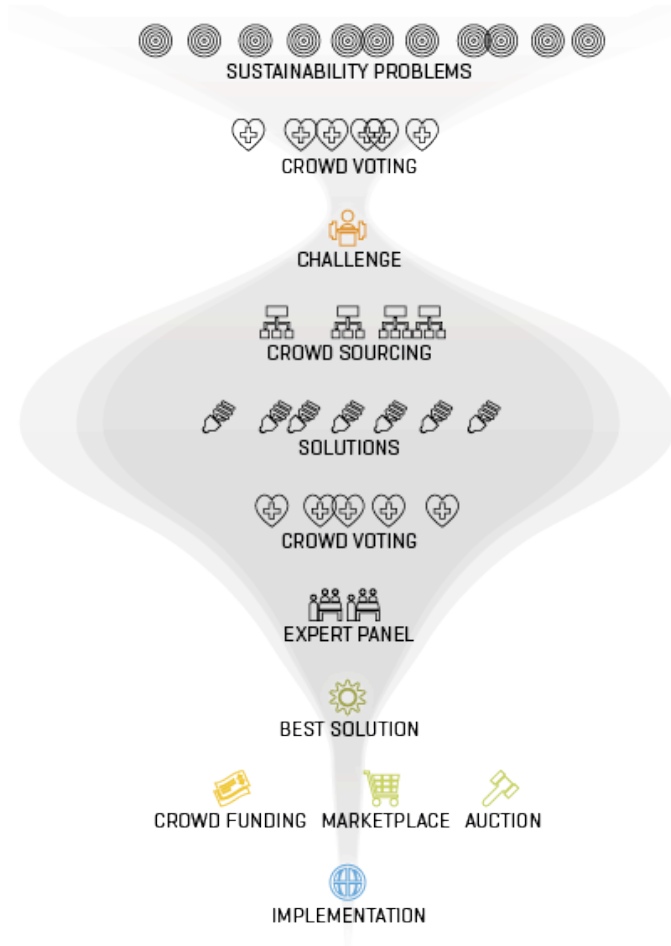

Figura 1 - Modelo de PDP via Crowd-Design.

Fonte: SuM (2014, web). 
O desafio consiste na definição de uma pergunta central, apoiada por informações que permitam aos participantes a compreensão adequada do problema. Estas informações podem incluir desde relatos textuais até vídeos síntese, storyboards ou fotografias. Neste caso, muitas poderão ser as ideias de solução para o desafio etapa solution. Por este motivo, ao final do processo de Crowd-Sourcing, as ideias de soluções recebidas são postas em votação, sendo este o segundo momento onde ocorre o Crowd-Voting. Este acontece a partir do envolvimento da comunidade de onde surgiu o problema juntamente com o envolvimento de um grupo de especialistas (expert panel).

Após a escolha da melhor ideia de solução do problema central do desafio etapa best solution -, inicia-se o processo de viabilização de implementação. Esta é realizada a partir do processo de Crowd-Funding, via de regra aberto, onde a ideia de solução escolhida é apresentada à multidão com o intuito de que a produção seja por ela financiada. A implementação do projeto ocorre quando a melhor ideia de solução é produzida com os recursos arrecadados (SuM, 2014, web). Pode-se utilizar também as plataformas de marketplace ou leilões virtuais para comercializar as soluções desenvolvidas.

\section{MÉTODO DE PESQUISA}

A coleta de dados nesta pesquisa vem sendo realizada com base nos princípios de Thiollent (1985), Mello e Turrioni (2011) e Robson (1993) para a pesquisa-ação. Para tanto, a pesquisa de campo segue, desta forma, um processo cíclico, sendo que o presente artigo foi elaborado quanto o processo de Crowd-Design se encontrava na etapa de levantamento de problemas, conforme ilustra a figura a seguir.

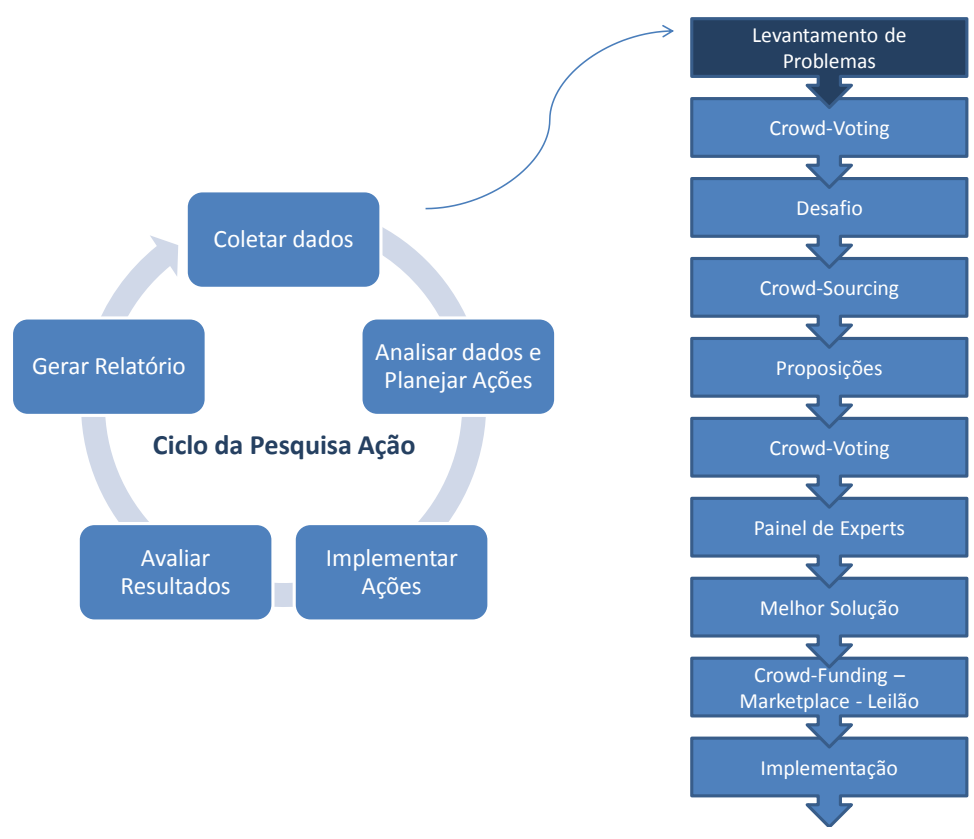

Figura 2: Ciclo da Pesquisa Ação e o Contexto da Fase do Projeto Sustainable Maker Fonte: Dos autores.

Na pesquisa-ação onde o modelo do Projeto Sustainability Maker vem sendo desenvolvido, além do envolvimento de uma comunidade de baixa renda no município de Piraquara/PR, há o envolvimento também de uma empresa da iniciativa privada. $O$ 
desafio do projeto é justamente a compatibilização da demandas e interesses da comunidade com os delineamentos estratégicos e as competências da empresa parceira.

A fase na qual se encontrava o projeto quando da redação do presente artigo é referente ao "levantamento de problemas". Para realizar esta etapa, o protocolo de coleta de dados prevê três visitas a campo: (1) reunião com a líder da comunidade para verificar o interesse em participar do projeto; (2) consulta preliminar acerca do perfil da comunidade (questionário) e levantamento inicial de problemas percebidos pelos moradores em resposta à pergunta "qual o principal problema em sua habitação?"; (3) coleta de dados em amostra de moradias através de múltiplas técnicas de coleta de dados (entrevista, paparazzi e storytelling). A estratégia de análise envolve a utilização de brainstorming, mapas mentais e storyboards.

\section{ANÁLISE COMPARATIVA DO MODELO ORTODOXO DO PDP x CROWD-DESIGN}

A seguir é apresentada análise comparativa do PDP e Crowd-Design com foco em cinco aspectos. A análise utiliza tanto informações oriundas da literatura assim como insights obtidos na pesquisa ação em andamento.

\subsection{Definição do Problema}

O modelo de Rozenfeld et al. (2006) utiliza uma estrutura modular, estabelecendo em seu primeiro módulo as definições estratégicas, a partir das quais é determinado o foco das próxima etapas do PDP. No modelo do Projeto Sustainainability Maker, a primeira etapa trata da compreensão do problema, o que pode incluir atividades como: (1) contatar a comunidade, (2) coletar suas contribuições e (3) fazer uma primeira triagem com relação aos problemas relatados. Só a partir daí é que os problemas poderão ser entendidos, votados (etapa Crowd-Voting), e o problema escolhido poderá ser transformado em desafio. Este modelo prioriza, portanto, o sentido "bottom up" do processo de decisão.

Segundo Manzini (2008), quando há mudanças no modo como indivíduos ou comunidades agem para resolver seus problemas ou criar novas oportunidades, há, na verdade, inovações guiadas por mudanças de comportamento dos indivíduos, geralmente emergindo através de processos operacionais "de baixo para cima" em vez daqueles "de cima para baixo" (MANZINI, 2008, p. 62). Os processos operacionais aos quais o autor se refere são, na verdade, iniciativas cuja a própria comunidade se envolve, desde a definição até a solução dos problemas. Conclui-se, portanto, que sob o ponto de vista da definição do problema, a abordagem em Crowd-Design oferece maiores possibilidades de resultar em soluções efetivamente sustentáveis pois amplia a governança do processo de Design.

Para o levantamento de problemas, a equipe da pesquisa-ação já realizou o encontro com os moradores da comunidade, onde além de apresentar o projeto, também foram coletados dados sobre, por exemplo, a quantidade média de moradores por habitação, materiais utilizados para a construção das mesmas e quantidade de cômodos. Porém, em resposta à pergunta "qual o principal problema em sua habitação?", alguns moradores citaram, por exemplo, a falta de identificação das ruas (como placas e número do CEP). Entende-se que a resolução a este tipo de problema compete aos órgãos públicos relacionados à urbanização. Nesse sentido, a coleta de dados em amostras de moradias vem se mostrando mais eficaz. Até a 
finalização deste artigo, esta coleta ainda não havia sido concluída. Porém, a partir dos dados já obtidos com a aplicação das técnicas de observação e storytelling, começam a aparecer os reais problemas, diretamente relacionados às habitações. Apesar disso, é apenas com a conclusão desta etapa que os dados serão analisados por completo para que se possa retornar à comunidade para a apresentação dos problemas comuns e para a realização do primeiro Crowd-Voting.

\subsection{Definição de briefing}

No modelo de Rozenfeld et al. (2006), a macro-fase denominada prédesenvolvimento contempla o Planejamento do Produto, que inclui a descrição do Briefing. Para Tavares (2007), Briefing é um instrumento usado para coletar informações para, com base nelas, planejar e agir. A equipe de Design é usualmente claramente definida e as possiblidades de comunicação com o público alvo é ampla, possibilitando inclusive a própria revisão do problema.

No modelo aberto da Plataforma Sustainability Maker (SuM, 2014, web), podese dizer que a definição do Briefing acontece na macro-fase denominada challenge, pois este é o momento de síntese das informações críticas que serão utilizadas pela "multidão" na elaboração de propostas para solução do problema (Crowd-Sourcing). Clareza no Briefing, além de linguagem estimulante, é um dos aspectos críticos para o sucesso de um desafio no processo de Crowd-Design. Para definir o Briefing, portanto, são necessários testes para saber se as informações contidas no mesmo são suficientes e entendíveis. Num processo de Crowd-Sourcing, o desenvolvimento da solução é aberto, isso significa que profissionais e pessoas de todo o mundo podem participar, enviando suas ideias. Nesse sentido, até mesmo a questão do idioma pode ser uma barreira para sua plena realização.

Assim, como forma de já testar qual a melhor maneira de disponibilizar as informações na plataforma do Projeto Sustainability Maker, foi desenvolvido um vídeo ${ }^{1}$ teaser, que traz o apontamento de um problema na visão de uma das moradoras da comunidade. O vídeo foi elaborado de maneira a contextualizar o tema - Habitação de Interesse Social; apresentar a comunidade através de imagens coletadas no local; e mostrar o depoimento da moradora. Para que o vídeo pudesse ser visto e entendido por um maior número de pessoas, o idioma utilizado nos textos explicativos e nas legendas foi o inglês.

No caso da geração de ideias a partir do Crowd-Sourcing, os autores Azzam e Jacobson (2011); Alonso (2012); Alonso e Mizzaro (2012) enfatizam a necessidade de realizar testes com a equipe interna antes de abrir o desafio para a participação da comunidade externa, pois é fundamental que as informações disponibilizadas no Briefing sejam claras e suficientes para que as pessoas possam entender o desafio de maneira correta e, consequentemente, gerar ideias de soluções que façam sentido.

Os usuários das comunidades são usualmente os que melhor conhecem o contexto e dinâmica do problema. Contudo, como observado na pesquisa-ação, muitas vezes as pessoas nestas comunidades não tem uma linguagem para comunicar seus problemas para a "multidão". O profissional Designer pode contribuir neste aspecto, utilizando a competências intrínsecas à área, bem como instrumentos de áreas como antropologia, psicologia e sociologia.

\footnotetext{
${ }^{1} \mathrm{O}$ vídeo está disponível para visualização neste link:

https://www.youtube.com/watch?v=5xQ9tDbsFqs\&feature=youtu.be
} 
Assim, no projeto de Crowd-Design em desenvolvimento, a definição do Briefing será realizada pela equipe após a primeira sessão de Crowd-Voting realizada na comunidade. O problema escolhido pelos moradores será, então, transformado em desafio. Os documentos disponibilizados na plataforma de Crowd-Sourcing ${ }^{2}$ deverão, além de contextualizar e esclarecer o problema, apresentar os resultados almejados com o projeto. Os materiais previstos para compor o Briefing do desafio são: textos explicativos, apresentando a comunidade e definições do escopo; imagens em foto e vídeo, bem como ilustrações como infográficos para ilustrar o contexto e a realidade da comunidade. Ainda, devido à parceria com a empresa privada, o Briefing deverá conter informações sobre aspectos relativos à produção da solução. Por este motivo, as sugestões de solução deverão vir acompanhadas de documentos que explicitem as informações técnicas de produção, inclusive para que possa ser entendido e replicado em contextos semelhantes. Esta replicação poderá ser facilitada pelos marketplaces e leilões virtuais.

\subsection{Processo de Desenvolvimento da Solução}

No modelo de Rozenfeld et al. (2006) as informações contidas no Projeto Informacional orientam a geração de soluções, iniciada com a fase do Projeto Conceitual. Nesta fase é onde acontecerá a busca, a criação, a representação e a seleção de soluções para o problema identificado. No caso do modelo da abordagem de Crowd-Design utilizada no Projeto Sustainability Maker a fase de desenvolvimento da solução é quando acontece a abertura do processo para o Crowd-Sourcing. Assim, as pessoas envolvidas nesta fase podem ser absolutamente desconhecidas do usuário, justamente por se encontrarem na multidão.

A abordagem metodológica que esta multidão de "Designers" utilizará no processo de criação e desenvolvimento das proposições de soluções é ampla e ilimitada. Para superar este obstáculo é possível que a plataforma de Crowd-Design possa determinar já no "challenge" (desafio) as ferramentas, estruturas e escopo mínimo das proposições, como, por exemplo:

- Explique sua ideia em uma sentença;

- Qual é a necessidade que você está querendo resolver?

- Quem se beneficiará da implementação de sua ideia?

- Quem vai monitorar seu sucesso?

- Quem está equipado para implementar sua ideia no mundo real? Você? Sua organização? Outra organização ou entidade?

- Onde esta ideia seria implementada?

- Como poderia ser prototipada ou testada sua ideia de forma a testar sua pressuposições?

Assim, prevê-se para o projeto em andamento a realização de um pré-teste junto à uma equipe interna formada por especialistas e não-especialistas para avaliar a consistência e a clareza das informações contidas no Briefing. Os feedbacks recebidos com a realização do pré-teste serão analisados e, caso seja necessário, serão realizadas as devidas alterações. Após o desafio ser disponibilizado definitivamente na plataforma de Crowd-sourcing, o processo de desenvolvimento da solução deverá see

\footnotetext{
${ }^{2}$ A plataforma www.innonatives.com é a plataforma que será utilizada pelo projeto Sustainability Maker, e está em fase de finalização.
} 
continuamente monitorado, não no sentido de controlar os participantes, mas, principalmente, no sentido de fornecer feedbacks, promovendo contínua motivação para a participação da multidão. Após o prazo estipulado para o desenvolvimento da solução, as propostas serão analisadas por um painel de especialistas, bem como pela empresa parceira, em aspectos relativos à qualidade, viabilidade de produção e implicações para a sustentabilidade, e levadas para a segunda sessão de Crowd-Voting na comunidade.

\subsection{Financiamento e Risco}

Nas metodologias de PDP ortodoxas, como é o caso da proposta por Rozenfeld et al. (2006), a implementação do projeto ocorre, geralmente, na penúltima fase, e antecede o monitoramento do desempenho do produto no mercado. Neste caso, a implementação do projeto ocorre ao final da macro-fase denominada desenvolvimento. Tendo em vista que este modelo já prevê a implementação do projeto desde a etapa de pré-desenvolvimento (quando da realização do planejamento estratégico), pode-se inferir que a prototipagem é garantida, já que a empresa prevê estes custos.

No modelo proposto por Rozenfeld et al. (2006), portanto, é a própria empresa que assume os riscos em conseguir novos consumidores para o produto. $O$ mesmo, porém, não acontece no modelo de PDP que visa o Crowd-Design. Neste modelo, a implementação do projeto pode ser considerada incerta, tendo em vista que, via de regra, depende do sucesso do crowdfunding (ou marketplace ou leilão) para efetivamente viabilizar financeiramente sua realização. Alternativas típicas de colaboração em um ambiente Crowd-Funding são exemplificadas abaixo, com base nas proposições iniciais geradas na pesquisa-ação:

- Ofereça R\$1 ou mais: HALL DA FAMA E COMUNIDADE: Seu nome irá para o Hall da Fama no site do projeto. Além disto você fará parte da comunidade de membros patrocinadores do projeto. Obrigado!

- Ofereça R\$15 ou mais: VIDEO: Seu nome será mostrado em um vídeo documentário que será feito para o Projeto onde apresentaremos um grande "obrigado" a todos os que colaborarem com nossa iniciativa.

- Ofereça R\$25 ou mais: CARTÃO SKETCH: Receba uma impressão em alta qualidade de sketches do projeto. Coloque isto na parede ou mesmo em uma moldura em sua mesa de trabalho que Ihe relembrará de sua contribuição ao projeto.

- Ofereça R\$35 ou mais: CAMISETA: Seja parte da gangue e ganhe uma camiseta com o slogan do Projeto (tamanhos $P, M$ e XG). A camiseta é produzida na cor branca com um print exclusivo que vai se diferenciar de suas outras camisetas.

- Ofereça R\$39 ou mais: BONÉ: Deixe todo mundo saber que você apoia um grande Projeto e que está utilizando um boné de alta qualidade;

- Ofereşa R\$75 ou mais: VISITE A COMUNIDADE: Encontre também o time de Designers e tire uma foto com um protótipo em um tour através da comunidade do projeto. Você receberá um Cartão Sketch para comemorar sua visita memorável;

- Ofereça R\$ 129 ou mais: BOLSA ESPECIAL: Este é um acessório de alta qualidade, para uso diário, desenhado pelo time do projeto;

- Ofereça R\$1.700 ou mais: adquira um dos Produtos desenvolvidos pelo Projeto com um Design especial para você. Ficará perfeito em sua casa ou apartamento, tornado seu ambiente único.

O Crowd-Funding é associado a uma gama de esperanças e ideais, para além do retorno econômico imediato. Em projetos que visam a sustentabilidade, o CrowdFunding é uma prática através da qual pode-se mobilizar os recursos necessários, mesmo que em pequena escala, para fornecer mais oportunidades para mais pessoas. 
Desta maneira, pode-se fomentar a produção popular mais difundida entre aqueles que não poderiam ter acesso ao capital inicial para financiar seus projetos criativos, reforçando os níveis de engajamento (BANNERMAN, 2013).

Para o projeto de Crowd-Design em andamento, prevê-se o aporte financeiro (integral ou parcial) da empresa parceira, diminuindo, desta forma, o risco de não concluir o projeto com um modelo/protótipo. Tendo em vista que esta empresa estará envolvida desde o início do processo até a escolha da "best solution", é provável que a empresa assuma os custos de produção do modelo. Este, por sua vez, poderá servir para a verificação do funcionamento in loco da solução.

\subsection{Propriedade intelectual/industrial}

Nas metodologias de PDP ortodoxas, a propriedade intelectual de um projeto tem conexão com a propriedade industrial, e por este motivo, requerem um processo muitas vezes demorado. Com a propriedade industrial é possível assegurar o monopólio ou o uso exclusivo sobre determinada criação ou inovação no mercado.

No processo de Crowd-Design o problema dos direitos autorais não pode ser ignorado, mesmo não sendo obrigatório. Assim, uma opção para projetos desenvolvidos em Crowd-Design e com foco na sustentabilidade é o lançamento de desafios em licença aberta. Um exemplo é o Creative Commons (CC), um tipo de licença jurídica gratuita. O CC é uma organização sem fins lucrativos, cujo objetivo é o compartilhamento e o uso da criatividade e do conhecimento. Disponibilizando o material em licença aberta, os autores dos projetos fornecem, de forma simples e padronizada, a concessão do direito de uso das suas obras intelectuais. O CC é uma estratégia eficaz para maximizar a criatividade, o compartilhamento e a inovação (CREATIVE COMMONS, 2014, web). O Projeto Sustainability Maker prevê, em seu escopo, a utilização do CC para a concessão do direito de produção dos projetos desenvolvidos nesta plataforma.

Assim, uma vez inserido na plataforma Sustainability Maker, os resultados do projeto de Crowd-Design em andamento poderão ser compartilhados com o mundo todo, através do marketplace formado com este objetivo. Nesse sentido, uma vez que a solução encontrada terá licença aberta em CC, muitas poderão ser as comunidades beneficiadas com a solução encontrada pelo projeto. No que tange à sustentabilidade, portanto, este fato pode ser considerado uma grande vantagem.

\section{CONSIDERAÇÕES FINAIS}

As plataformas para Crowd-Design constituem em oportunidade de canalizar a criatividade da população de baixa renda no encaminhamento dos problemas de seu próprio entorno. O reconhecimento desta oportunidade ocorre em um contexto onde esta população vem aumentando gradualmente sua capacidade de compra. Este aumento de capacidade, ao mesmo tempo que traz benefícios na elevação da qualidade de vida da população de baixa renda, traz consigo grande risco ambiental devido à possibilidade de réplica dos estilos de vida da população mais rica. Por outro, lado este mesmo fenômeno oferece a possibilidade do design, via este novo ambiente democrático de projeto instrumentalizado pela internet, possibilitar o "leap-frog" nos padrões de consumo (descontinuidade sistêmica), ou seja, a adoção de soluções radicalmente superiores sob a ótica da sustentabilidade. 
Do mesmo modo, a aplicação do Crowd-Design para a sustentabilidade pode ser potencializada com o envolvimento, desde o início, de empresas parceiras que visam, sobretudo, a inovação em seus processos produtivos. O desafio, porém, está na compatibilização da estratégia da empresa com os problemas demandados pela comunidade. Nesse sentido, alguns dos problemas já apontados pela comunidade terão que ser descartados, não só pela sua natureza (como o exemplo dado), mas principalmente por não estarem alinhados com as competências e estratégias da empresa parceira ao projeto..

Contudo, apesar do rápido crescimento desta modalidade de projeto/produção, não se observa também registros de sua efetiva adoção no ambiente de sala de aula em Cursos de Design. O ambiente crowd exige novas metodologias de projeto e nova formas de relacionamento do Designer com todos os atores associados ao desenvolvimento e implementação de soluções, incluindo o próprio usuário. Desta forma, entende-se como premente ampliar a compreensão acerca de métodos e ferramentas associados ao Crowd-Design de forma a permitir que este conteúdo seja passível de inserção no processo de aprendizado dos graduandos dos Cursos de Design.

\section{AGRADECIMENTOS}

Os autores agradecem o apoio financeiro da CAPES (Coordenação de Aperfeiçoamento de Pessoal de Nível Superior) pela bolsa de doutorado demanda social.

\section{REFERÊNCIAS}

ABRAHAMSON, Shaun; RYDER, Peter; UNTERBERG, Bastian. Crowdstorm: the future of innovation, ideas and problem solving. New Jersey: John Wiley \& Sons, Inc., Hoboken, 2013.

ADAMS, Samantha A. Sourcing the crowd for health services improvement: The reflexive patient and "share-your-experience" websites. In: Social science \& medicine, v. 72, n. 7, p. 1069-1076, 2011.

ALBORS, J; RAMOS, J. C.; HERVAS, J. L. New learning network paradigms: Communities of objectives, Crowd-Sourcing, wikis and open source. In: International Journal of Information Management, v. 28, n. 3, p. 194-202, 2008.

ALONSO, O. Implementing Crowd-Sourcing-based relevance experimentation: an industrial perspective. Information Retrieval, v. 16, n. 2, p. 101-120, 2012. Disponível em: <http://link.springer.com/10.1007/s10791-012-9204-1>. Acesso em: 9/4/2014.

ALONSO, O.; MIZZARO, S. Using Crowd-Sourcing for TREC relevance assessment. Information Processing \& Management, v. 48, n. 6, p. 1053-1066, 2012. Disponível em: <http://linkinghub.elsevier.com/retrieve/pii/S0306457312000052>. Acesso em: 24/3/2014.

ALSEVER, Jennifer. What is Crowd-Sourcing? 2008. Disponível em: <http://www.cbsnews.com/news/what-is-Crowd-Sourcing/> Acesso em: 28/03/2014. AZZAM, T.; JACOBSON, M. R. Finding a Comparison Group: Is Online Crowd-Sourcing a Viable Option? In: American Journal of Evaluation, v. 34, n. 3, p. 372-384, 2011.

BANNERMAN, S. Crowdfunding Culture. In: Journal of Mobile Culture, v. 7, n. 1, p. 0130, 2013.

BAXTER, M. Projeto de produto: guia prático para o desenvolvimento de novos 
produtos. São Paulo: Edgard Blücher, 2000.

BELLEFLAMME, Paul; LAMBERT, Thomaz; SCHWIENBACHER, Armin. Crowdfunding: Tapping the Right Crowd. In: ECORE International Association for Research and Teaching, 2011. Disponível em: http://www.ecore.be/DPs/dp_1310721784.pdf Acesso em: 27/04/2014.

CREATIVE COMMONS. Licenças. Disponível em: http://creativecommons.org/licenses/. Acesso em 28/03/2014.

CRUL, M., DIEHL, J. C. Design for sustainability: a practical approach for developing economies. Paris: United Nations Environmental Programme, Technical University Delft, 2006.

GOMES, Nivaldo Simões. Proposta de Critérios para Seleção de Ferramentas da Dimensão Ambiental do Design Sustentável. Dissertação de Mestrado em Design. Programa de Pós-Graduação em Design da UFPR, 2011.

NEEDEL, Steve. Meet The New Consumer. 2013. Disponível em: <http://www.greenbookblog.org/2013/09/11/meet-the-new-consumer/> Acesso em: 28/03/2014.

PEREIRA, A. F. Da sustentabilidade ambiental e da complexidade sistêmica no design industrial de produtos. In: Estudos em Design, Rio de Janeiro, v. 10, n. 1, p. 37-61, 2003

PRIVE, Tanya. What Is Crowdfunding And How Does It Benefit The Economy. 2012. Disponível em: <http://www.forbes.com/sites/tanyaprive/2012/11/27/what-iscrowdfunding-and-how-does-it-benefit-the-economy/> Acesso em: 29/03/2014.

ROBSON, C. Real world research: a resource for social scientists and practitioner. Oxford: Blackwell, 1993.

ROZENFELD, H.; FORCELLINI, F.A.; AMARAL, D.C.; TOLEDO, J.A.; SILVA, S. ALLIPRANDINI, D.H.; SCALICE, R.K. Gestão de desenvolvimento de produtos: uma referência para a melhoria do processo. São Paulo: Saraiva, 2006.

SANTOS, Onélio Luis S. Considerações sobre a propriedade intelectual no processo de globalização mundial e integração regional, com uma sucinta abordagem sobre sua proteção no Mercosul e no Brasil. Jus Navigandi, Teresina, ano 6, n. 52, 1 nov. 2001. Disponível em: <http://jus.com.br/artigos/2436>. Acesso em: 27/04/2014.

SUSTAINABILITY MAKER PLATFORM. Disponível em: <http://sustainabilitymaker.org/>. Acesso em: 30/03/2014.

TAPSCOTT, D. \& WILLIAMS, A. D. Wikinomics: How Mass Collaboration Changes Everything. Portfolio Hardcover; Expanded edition, April 17, 2008.

TAVARES, Maurício. Comunicação empresarial e planos de comunicação: integrando teoria e prática. São Paulo: Atlas, 2007.

THIOLLENT, M. Metodologia da pesquisa-ação. 15. ed. São Paulo: Cortez, 2007.

TURRIONI, J. B.; MELLO, C. H. P. Apostila de Metodologia de Pesquisa em Engenharia de Produção.Itajubá: Universidade Federal de Itajubá, 2011.

VARŽINSKAS, V.; GURAUSKIENE, I.; PIPINYTE, L.. Improvement in enviromental performance of vapour compression refrigeration system by the model for environmental product development. In Environmental research, engineering and management $=$ Aplinkos tyrimai, inžinerija ir vadyba. ISSN 1392-1649. nr. 2(40), p. 5969, 2007. 